\title{
PEDOT:PSS Overcoating Layer for Mechanically and Chemically Stable Ag Nanowire Flexible Transparent Electrode
}

\author{
Byungil Hwang ${ }^{1}$ and Sooman Lim $^{2}$ \\ ${ }^{1}$ BASF Electronic Materials R\&D Center Asia, Suwon 16419, Republic of Korea \\ ${ }^{2}$ Graduate School of Flexible and Printable Electronics, Chonbuk National University, Jeonju 54896, Republic of Korea \\ Correspondence should be addressed to Sooman Lim; sooman.lim@gmail.com
}

Received 22 January 2017; Revised 5 March 2017; Accepted 12 March 2017; Published 6 April 2017

Academic Editor: Albert Nasibulin

Copyright (c) 2017 Byungil Hwang and Sooman Lim. This is an open access article distributed under the Creative Commons Attribution License, which permits unrestricted use, distribution, and reproduction in any medium, provided the original work is properly cited.

\begin{abstract}
We investigated the effect of poly(3,4-ethylenedioxythiophene)-poly(styrenesulfonate) (PEDOT:PSS) deposition on the chemical and mechanical stability of Ag nanowire flexible electrodes. A large number of bending cycles, up to 500,000 cycles, were imposed on the Ag nanowire electrodes with and without PEDOT:PSS overcoating layer. In situ resistance measurement during bending tests revealed that the Ag nanowire electrode with PEDOT:PSS overcoating layer was mechanically reliable, showing a $21.9 \%$ increase in resistance after 500,000 cycles of bending. Scanning electron microscope images revealed that the failure of the Ag nanowire network occurred along with cracks initiated in the PEDOT:PSS layer, which resulted in the increase in resistance under bending. Furthermore, the PEDOT:PSS deposition enhanced the chemical stability of Ag nanowire electrode, which showed no significant increase in resistance after exposure in air for 50 days. Our study underscored that PEDOT:PSS is effective in protecting the Ag nanowires, while maintaining the high mechanical stability.
\end{abstract}

\section{Introduction}

Technological advances in flexible/wearable electronics require the development of robust electrodes that can tolerate severe mechanical bending [1-7]. The current choice for transparent electrodes is indium tin oxide (ITO) film, but the brittleness and the high processing temperature of ITO film make its use for flexible/stretchable electronics difficult $[8,9]$. In addition, the shortage of indium reserves is continuously increasing material costs. Therefore, there have been several researches to develop novel materials to replace ITO. The potential alternatives to replace ITO are carbon nanotubes (CNT) [10, 11], graphene [12, 13], and metal nanowires [14-16]. Among the metal nanowires, Ag nanowire network is the most attractive candidate, owing to its high electrical conductivity and excellent optical transmittance [17-21]. Furthermore, Ag nanowire network is known to have outstanding mechanical flexibility, which is suitable for flexible/wearable electronics $[2,7,22]$.

However, a limitation of $\mathrm{Ag}$ nanowires is their poor chemical stability on exposure to air $[6,19,23]$. The large surface-to-volume ratio allows the Ag nanowires to be easily oxidized when exposed to air, thereby degrading the electrical conductivity of the Ag nanowire network $[2,23]$. Therefore, a suitable overcoating layer is required to prevent the $\mathrm{Ag}$ nanowires from surface oxidation; metal oxide $[1,3,24]$, graphene [2, 6], and polymeric layer [25-27] are examples of the overcoating layers. PEDOT:PSS has been widely studied for the protective layer on Ag nanowires, due to its high conductivity and optically transparent property [25-27]. For example, Chen et al. reported that adopting the PEDOT:PSS reduced the sheet resistance of Ag nanowire network, without a significant loss of optical transparency [25]. In addition, the Ag nanowire with PEDOT:PSS overcoating layer was stable under ambient moisture, without significant loss of electrical conductivity [25].

However, since the main goal of the Ag nanowire network is its use for flexible electronics, understanding of the mechanical reliability of the Ag nanowire networks with PEDOT:PSS layer is crucial. To satisfy the requirements for reliability in the actual operational conditions of electronic devices, the conductor should be able to withstand a bending 
strain of more than 300,000 cycles [7]. But no systematic studies have previously been conducted on the mechanical reliability of Ag nanowire network with PEDOT:PSS layer under large cycles of bending.

This work systematically investigated the effects of PEDOT:PSS layer on the mechanical and chemical stability of Ag nanowire network. Ag nanowires on a flexible polyethylene terephthalate (PET) substrate were coated with PEDOT:PSS by using a spin coater. By imposing a large number of bending cycles, 500,000 cycles, while measuring the resistance in situ, the mechanical reliability of the $\mathrm{Ag}$ nanowire with PEDOT:PSS layer was systematically evaluated. The deformation behavior of Ag nanowire with PEDOT:PSS layer was further confirmed by analyzing the microstructure before and after bending using scanning electron microscopy (SEM). In addition, the Ag nanowires with and without PEDOT:PSS layer were exposed to air for 50 days, to confirm the enhanced chemical stability from the PEDOT:PSS layer.

\section{Experiments}

2.1. Fabrication and Characterization of Ag Nanowire Electrode with PEDOT:PSS Layer. The Ag nanowires were synthesized by using a well-known polyol process. The details of the synthesizing method can be seen in [16]. The Ag nanowire solution was deposited on a flexible PET substrate by doctor blading. The height of the blade was fixed as $150 \mu \mathrm{m}$, and the blading speed was $10 \mathrm{~mm} / \mathrm{sec}$. The as-coated Ag nanowire electrodes were then annealed at $120^{\circ} \mathrm{C}$ for $10 \mathrm{~min}$ in air. The sheet resistance of the $\mathrm{Ag}$ nanowire electrodes was $15.1 \pm 1.3 \Omega / \square$, and the optical transmittance was $86.1 \pm$ $0.7 \%$. PEDOT:PSS aqueous solution with the solid content of $\sim 1.3 \mathrm{wt} \%$ (PH1000, Clevios ${ }^{\mathrm{TM}}$ ) was used in the study. The PEDOT:PSS was coated on the Ag nanowire electrodes by a spin coater. The spin-coating time and rpm were fixed at 30 seconds and 5,000 rpm, respectively. The PEDOT:PSS coated $\mathrm{Ag}$ nanowire electrodes were baked at $120^{\circ} \mathrm{C}$ for $5 \mathrm{~min}$ in air. The thickness of the PEDOT:PSS layer on Ag nanowire electrodes was measured as $200 \mathrm{~nm}$. PEDOT:PSS deposition decreased the sheet resistance of $\mathrm{Ag}$ nanowire electrode to $11.6 \pm 0.4 \Omega / \square$ and the optical transmittance to $83.8 \pm 1.1 \%$. The continuous PEDOT:PSS film was confirmed to increase the sheet resistance of the Ag nanowire electrodes while decreasing the optical transmittance. The optical transmittance was measured by UV-Vis spectrometry (Haze-Gard I) from BYKGardner Additives \& Instruments. Four-point probe (FPP2400, Dasol Engineering Co., Ltd.) was used to measure the sheet resistance of the Ag nanowire electrodes. The surface morphology analysis was performed by field-emission SEM (FE-SEM, Phillips, XL30 ESEM-FEG).

2.2. Bending Fatigue Test. Cyclic bending fatigue tester (CK Trading Co. Ltd.) was used to perform the cyclic bending tests on the Ag nanowire electrode with and without PEDOT:PSS layer (Figure 1). A detailed explanation of the bending fatigue tester can be found in [7]. In brief, the Ag nanowire electrodes were mounted on two plates using screw bolts, and $\mathrm{Cu}$
TABLE 1: Sheet resistance change of Ag nanowire electrodes with and without PEDOT:PSS overcoating layer as a function of bending cycles.

\begin{tabular}{lccccc}
\hline Cycle number & 0 & 1000 & 10,000 & 100,000 & 500,000 \\
\hline Without PEDOT (ohm) & 15.0 & 15.4 & 16.0 & 17.1 & 18.3 \\
With PEDOT (ohm) & 11.7 & 12.7 & 13.2 & 13.8 & 14.2 \\
\hline
\end{tabular}

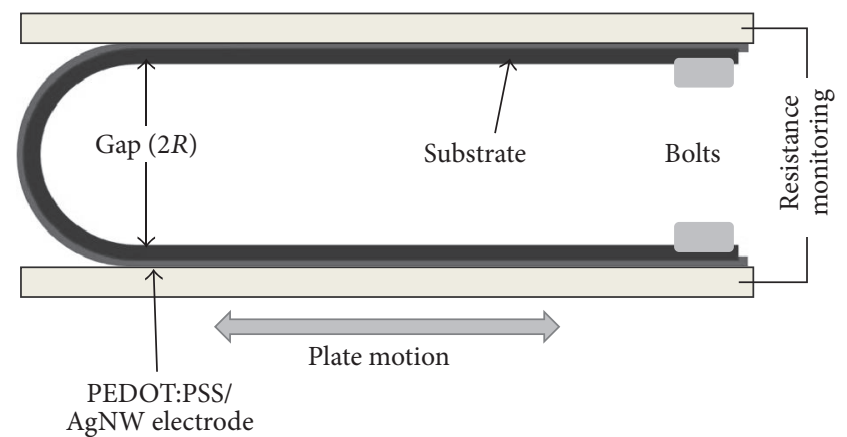

FIGURE 1: Schematic of cyclic bending fatigue tester.

pads at the end of each plate where the Ag nanowires were in contact enabled in situ resistance monitoring. The horizontal movement of the lower plate imposed repeated bending strain on the specimens. The bending strain could be calculated using the equation $\varepsilon=y / R$, where $y$ is the distance from the neutral plane and $R$, the radius, is the half value of the distance between the plates. Here, the gap between the two plates was fixed as $6 \mathrm{~mm}$, which corresponds to a bending strain of $2.1 \%$. The bending speed was 300 cycles $/ \mathrm{min}$, and the total number of bending cycles was 500,000 cycles.

\section{Results and Discussion}

3.1. Mechanical Reliability of Ag Nanowire Electrode with PEDOT:PSS Layer. To investigate the effect of the PEDOT:PSS layer on the mechanical reliability of Ag nanowire electrode, a large number of bending cycles, 500,000 cycles, were imposed on the Ag nanowire electrode with and without PEDOT:PSS layer. The Ag nanowire electrodes were prepared to have an initial sheet resistance of $\sim 15.1 \Omega / \square$, which was then decreased to $\sim 11.6 \Omega / \square$ after coating the PEDOT:PSS layer. Figure 2(a) shows the cyclic bending test results of bare Ag nanowire electrode and PEDOT:PSS coated Ag nanowire electrode. The result of Ag thin film with $100 \mathrm{~nm}$ thickness tested under the same testing condition was presented together for comparison. The mechanical reliability of the Ag nanowire electrode and the PEDOT:PSS coated Ag nanowire electrode was excellent compared to that of Ag thin film. The increase in fractional resistance of $\mathrm{Ag}$ thin film was $385.2 \%$ after 500,000 cycles of bending, while the Ag nanowire electrode and the PEDOT:PSS coated Ag nanowire electrodes showed $20.8 \%$ and $21.9 \%$ increase, respectively. The corresponding sheet resistance change of the Ag nanowire electrodes with and without PEDOT:PSS overcoating layer as a function of bending cycles can be seen in Table 1 .

The excellent mechanical reliability of the Ag nanowire networks is known to be due to their nanoscale dimension 


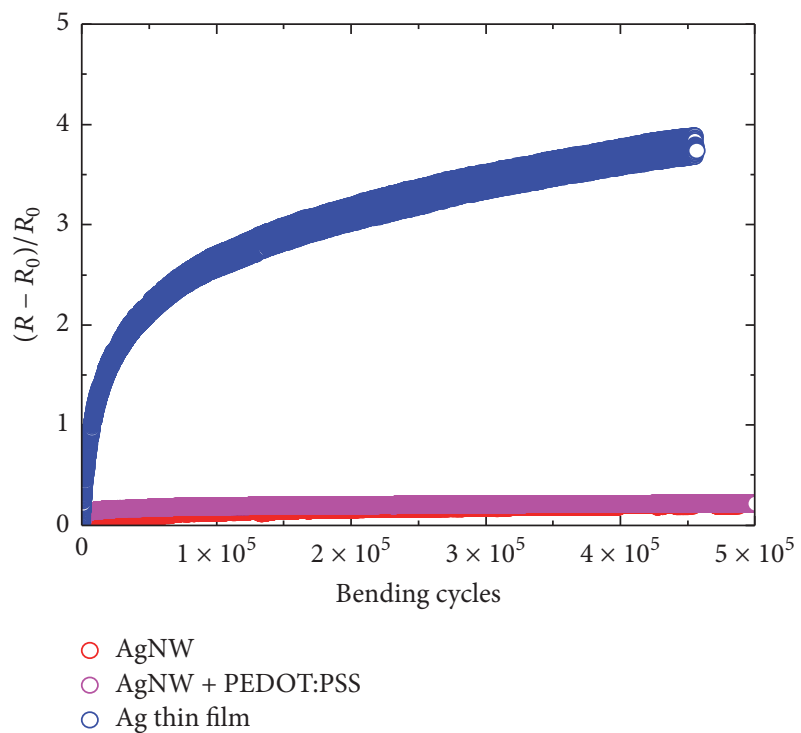

(a)

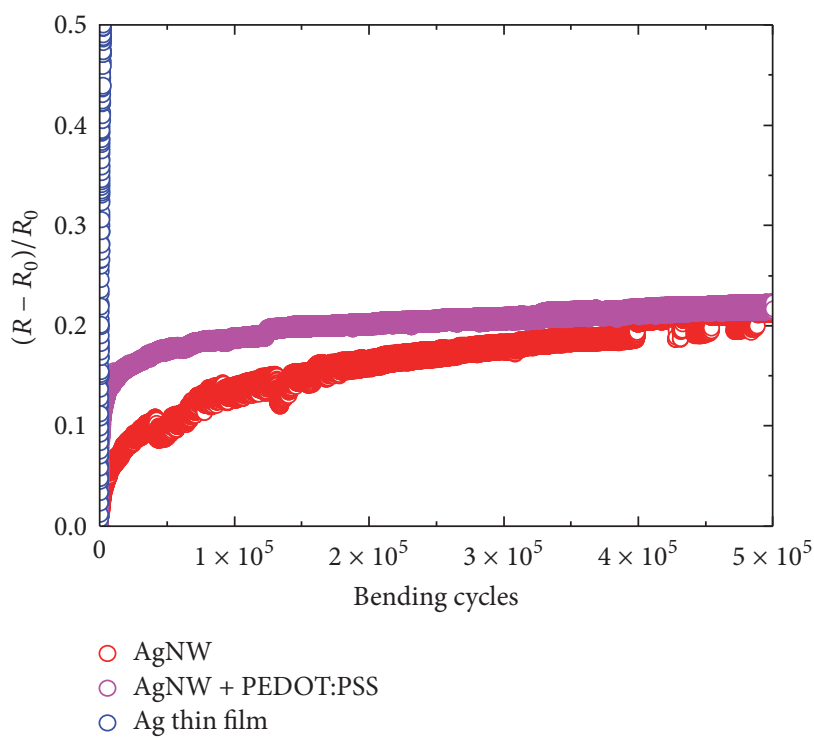

(b)

FIGURE 2: (a) Cyclic bending test results of the different specimens and (b) rescaled results of (a) within 50\% of fractional resistance change.

and the geometrical advantage of the network [2, 7, 22]. In case of the Ag thin film, the dislocation accumulation at the film/substrate interfaces during cyclic bending causes extrusion formation, where the cracks are initiated and propagated as the bending cycles increase further [7]. The cracks can act as the obstacle for the electrical current flow, which increases resistance. On the other hand, the size-dependent plasticity and the low density of surface defects observed in metal nanowires result in high strength that approached the theoretical strength, which is defined as $E / 10$ [2]. Therefore, the individual nanowire with high strength can bear more bending strain than can the bulk material. In addition to the high strength, the network geometry is a reason for the excellent mechanical stability of Ag nanowire electrodes. Under bending or stretching condition, the nanowire network can release the imposed bending strain, by adjusting its network along the direction of deformation [2, 7, 22]. Furthermore, the dislocation accumulation observed in the thin films is difficult to occur in the network geometry [2, $7,22]$, which can be another reason for the enhancement of mechanical reliability. Although the PEDOT:PSS layer has a continuous blanket structure, the high flexibility of PEDOT:PSS film [28, 29] made the Ag nanowire network sustain its excellent mechanical reliability; thus, the gap of increase in fractional resistance between the nanowire networks with and without PEDOT:PSS layer was small after 500,000 cycles (Figure 2(b)).

To further confirm the effect of PEDOT:PSS layer on the deformation behavior of Ag nanowire electrode, the surface morphology was analyzed for the bare Ag nanowire electrode and the PEDOT:PSS coated Ag nanowire electrode using SEM. Figure 3 presents the SEM imagery of each specimen before and after cyclic bending tests. The bare Ag nanowire electrode showed network structure (Figure 3(a)); Figure 3(d) shows that, after coating with PEDOT:PSS, the network structure was buried in the PEDOT:PSS film. After 1,000 cycles of bending, a number of failures of the individual Ag nanowires were observed (Figure 3(b)), while the cracks were initiated in the PEDOT:PSS layer, along which the $\mathrm{Ag}$ nanowires were also broken together (Figure 3(e)). Once the damage (such as breakage of $\mathrm{Ag}$ nanowire or cracks in PEDOT:PSS layer) initiated in the electrodes, the applied stress could be concentrated near the damaged area, which resulted in the propagation of the damage, consequently causing the detaching of a part of the conductive layer (Figures 3(c) and 3(e)). Since there was no current path in the damaged area where the conductive layer was removed, the resistance would increase rapidly as the fraction of damaged area increased.

3.2. Ambient Stability of Ag Nanowire Electrode with PEDOT:PSS Layer. The enhancement of chemical stability of Ag nanowire electrode by adopting a PEDOT:PSS layer was investigated by exposing the electrodes to ambient air for 50 days. The results of sheet resistance change depending on the exposure time are shown in Figure 4. While the sheet resistance of bare $\mathrm{Ag}$ nanowire electrode was remarkably increased from $\sim 15.2$ to $\sim 598.8 \Omega / \square$ after exposure in air for 50 days, there was no significant increase in sheet resistance of the Ag nanowire electrode with PEDOT:PSS layer. The SEM imagery taken for each specimen after exposure for 50 days shows that the oxide particle covered the surface of the bare Ag nanowires electrodes (Figure 5(a)), but the Ag nanowire electrode with PEDOT:PSS layer showed no observable oxidation (Figure 5(b)). The PEDOT:PSS layer was effective in preventing the $\mathrm{Ag}$ nanowire electrodes from contact with the oxygen or water in air, thereby suppressing surface oxidation. Surface oxidation was known to deteriorate the electrical conductivity of $\mathrm{Ag}$ nanowires, due to the composition change from $\mathrm{Ag}$ to $\mathrm{Ag}_{2} \mathrm{O}_{3}$ or $\mathrm{Ag}_{2} \mathrm{~S}$ as well as due to the reduced crosssectional area by oxidation [2]. Therefore, the suppressed surface oxidation observed in the Ag nanowire electrode with 


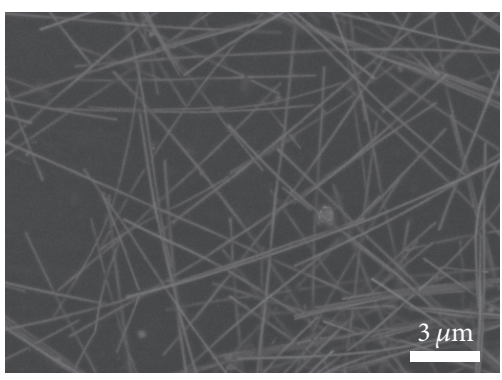

(a)

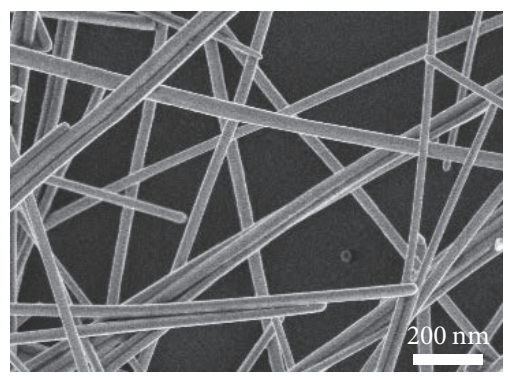

(d)

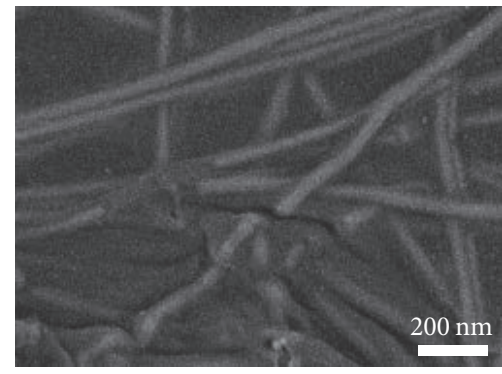

(b)

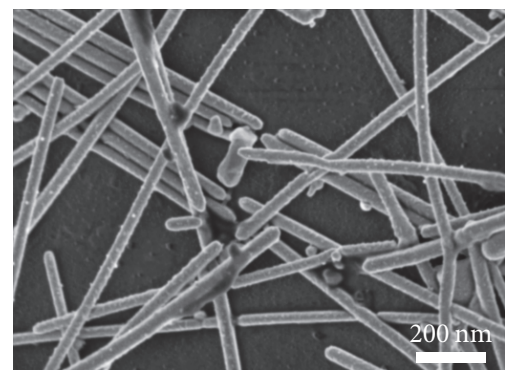

(e)

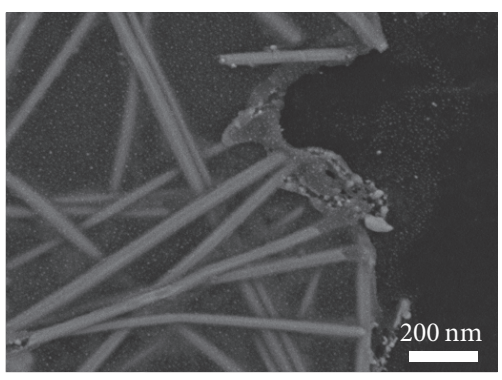

(c)

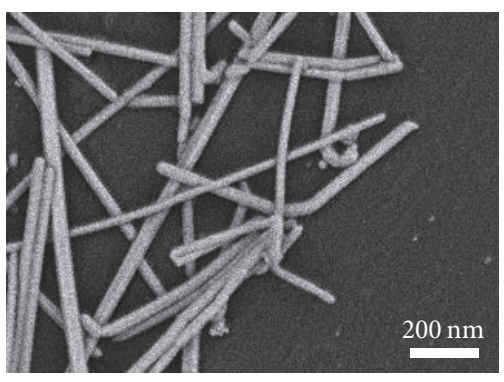

(f)

FIGURE 3: SEM images of $(\mathrm{a}-\mathrm{c})$ Ag nanowire electrodes with PEDOT:PSS overcoating layer and (d-f) bare Ag nanowire electrodes as a function of bending cycles: (a, d) before bending, (b, e) after 1,000 cycles, and (c, f) after 500,000 cycles.

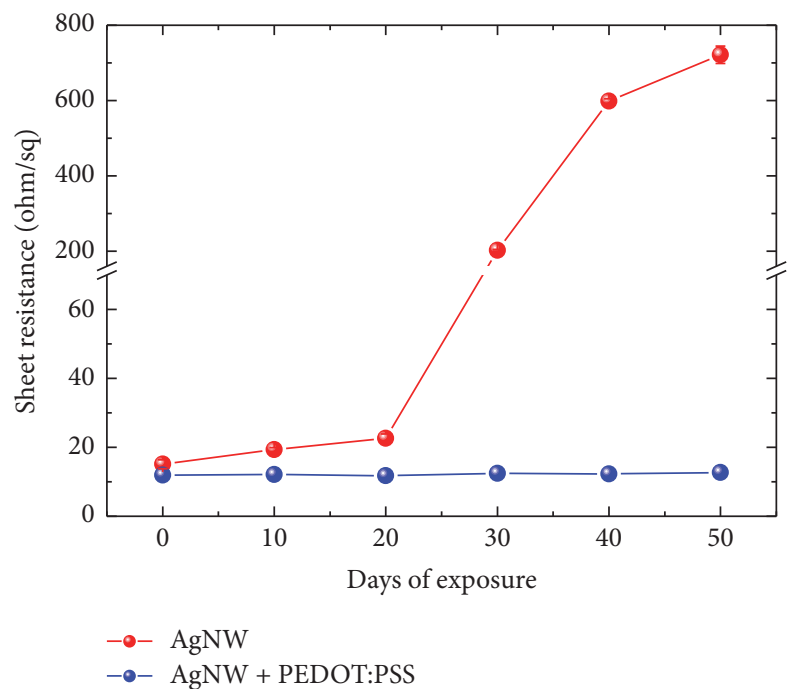

FIGURE 4: Sheet resistance change of Ag nanowire electrodes with and without PEDOT:PSS layer as a function of exposure time to air at room temperature.

PEDOT:PSS is in agreement with the lack of change in sheet resistance after exposure in air for 50 days.

\section{Conclusion}

In summary, the bending fatigue behavior of PEDOT:PSS coated Ag nanowire electrodes was explored by using the cyclic bending tester, which is capable of imposing a large number of cycles of bending up to 500,000 cycles when monitoring the resistance in situ. The cyclic bending fatigue tests

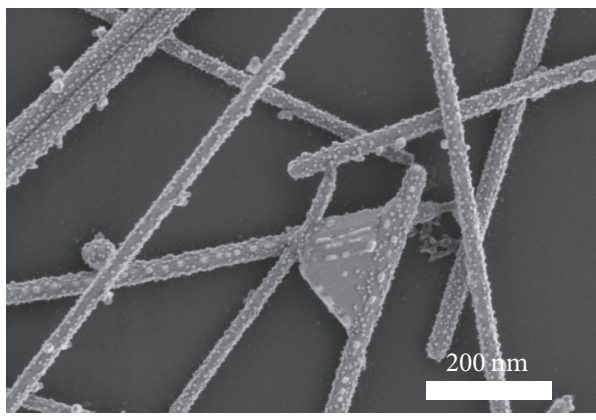

(a)

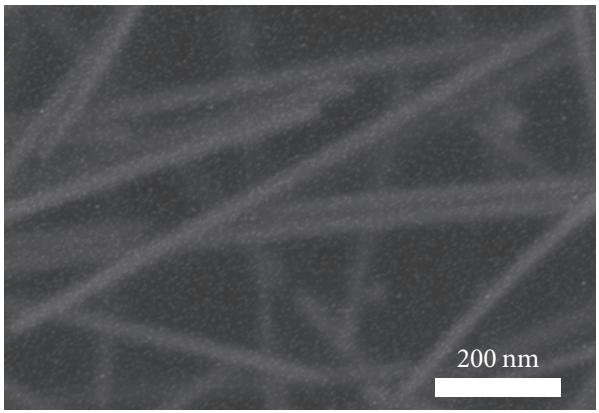

(b)

FIGURE 5: SEM images of Ag nanowire electrodes (a) with and (b) without PEDOT:PSS layer after exposing it to air for 50 days.

revealed that the Ag nanowire electrode with PEDOT:PSS layer was highly flexible and could sufficiently withstand the high number of cycles of bending. The increase in fractional resistance of the PEDOT:PSS coated Ag nanowire 
electrodes was $\sim 19$ times smaller than that of $\mathrm{Ag}$ thin film. Furthermore, the ambient stability of Ag nanowire electrodes was remarkably enhanced by adopting the PEDOT:PSS layer, showing no increase in sheet resistance after exposure in air for 50 days. The PEDOT:PSS was effective in protecting the Ag nanowires from surface oxidation, by preventing the Ag nanowire from contact with the oxygen or water in air. Our study highlighted that PEDOT:PSS is a suitable candidate The results of the deformation behavior of the Ag nanowire electrodes with PEDOT:PSS layer will provide practical guidance for applications of a hybrid system for flexible/wearable electronics.

\section{Conflicts of Interest}

The authors declare that there are no conflicts of interest regarding the publication of this paper.

\section{References}

[1] T.-B. Song, Y. S. Rim, F. Liu et al., "Highly robust silver nanowire network for transparent electrode," ACS Applied Materials and Interfaces, vol. 7, no. 44, pp. 24601-24607, 2015.

[2] B. Hwang, M. Park, T. Kim, and S. M. Han, "Effect of RGO deposition on chemical and mechanical reliability of Ag nanowire flexible transparent electrode," RSC Advances, vol. 6, no. 71, pp. 67389-67395, 2016.

[3] A. Kim, Y. Won, K. Woo, C.-H. Kim, and J. Moon, "Highly transparent low resistance $\mathrm{ZnO} / \mathrm{Ag}$ nanowire/ $\mathrm{ZnO}$ composite electrode for thin film solar cells," ACS Nano, vol. 7, no. 2, pp. 1081-1091, 2013.

[4] W. J. Scheideler, J. Smith, I. Deckman, S. Chung, A. C. Arias, and V. Subramanian, "A robust, gravure-printed, silver nanowire/metal oxide hybrid electrode for high-throughput patterned transparent conductors," Journal of Materials Chemistry C, vol. 4, no. 15, pp. 3248-3255, 2016.

[5] L. Hu, H. Wu, and Y. Cui, "Metal nanogrids, nanowires, and nanofibers for transparent electrodes," MRS Bulletin, vol. 36, no. 10, pp. 760-765, 2011.

[6] D. Lee, H. Lee, Y. Ahn, Y. Jeong, D.-Y. Lee, and Y. Lee, "Highly stable and flexible silver nanowire-graphene hybrid transparent conducting electrodes for emerging optoelectronic devices," Nanoscale, vol. 5, no. 17, pp. 7750-7755, 2013.

[7] B. Hwang, H.-A. Shin, T. Kim, Y.-C. Joo, and S. M. Han, "Highly reliable Ag nanowire flexible transparent electrode with mechanically welded junctions," Small, vol. 10, no. 16, pp. 33973404, 2014.

[8] Z. Chen, B. Cotterell, and W. Wang, "The fracture of brittle thin films on compliant substrates in flexible displays," Engineering Fracture Mechanics, vol. 69, no. 5, pp. 597-603, 2002.

[9] K. A. Sierros, N. J. Morris, K. Ramji, and D. R. Cairns, "Stresscorrosion cracking of indium tin oxide coated polyethylene terephthalate for flexible optoelectronic devices," Thin Solid Films, vol. 517, no. 8, pp. 2590-2595, 2009.

[10] K. S. Kim, Y. Zhao, H. Jang et al., "Large-scale pattern growth of graphene films for stretchable transparent electrodes," Nature, vol. 457, no. 7230, pp. 706-710, 2009.

[11] J. Wu, M. Agrawal, H. A. Becerril et al., "Organic light-emitting diodes on solution-processed graphene transparent electrodes," ACS Nano, vol. 4, no. 1, pp. 43-48, 2010.
[12] M. W. Rowell, M. A. Topinka, M. D. McGehee et al., "Organic solar cells with carbon nanotube network electrodes," Applied Physics Letters, vol. 88, no. 23, Article ID 233506, 2006.

[13] D. Zhang, K. Ryu, X. Liu et al., "Transparent, conductive, and flexible carbon nanotube films and their application in organic light-emitting diodes," Nano Letters, vol. 6, no. 9, pp. 1880-1886, 2006.

[14] J.-Y. Lee, S. T. Connor, Y. Cui, and P. Peumans, "Solutionprocessed metal nanowire mesh transparent electrodes," Nano Letters, vol. 8, no. 2, pp. 689-692, 2008.

[15] S. De, T. M. Higgins, P. E. Lyons et al., "Silver nanowire networks as flexible, transparent, conducting films: extremely high DC to optical conductivity ratios," ACS Nano, vol. 3, no. 7, pp. 1767$1774,2009$.

[16] T. Kim, A. Canlier, G. H. Kim, J. Choi, M. Park, and S. M. Han, "Electrostatic spray deposition of highly transparent silver nanowire electrode on flexible substrate," ACS Applied Materials and Interfaces, vol. 5, no. 3, pp. 788-794, 2013.

[17] Y. Wang, T. Feng, K. Wang, M. Qian, Y. Chen, and Z. Sun, "A facile method for preparing transparent, conductive, and paperlike silver nanowire films," Journal of Nanomaterials, vol. 2011, Article ID 935218, 5 pages, 2011.

[18] Y.-J. Song, J. Chen, J.-Y. Wu, and T. Zhang, "Applications of silver nanowires on transparent conducting film and electrode of electrochemical capacitor," Journal of Nanomaterials, vol. 2014, Article ID 193201, 7 pages, 2014.

[19] Y.-J. Tsai, C.-Y. Chang, Y.-C. Lai, P.-C. Yu, and H. Ahn, "Realization of metal-insulator transition and oxidation in silver nanowire percolating networks by terahertz reflection spectroscopy," ACS Applied Materials \& Interfaces, vol. 6, no. 1, pp. 630-635, 2014.

[20] S. B. Sepulveda-Mora and S. G. Cloutier, "Figures of merit for high-performance transparent electrodes using dip-coated silver nanowire networks," Journal of Nanomaterials, vol. 2012, Article ID 286104, 7 pages, 2012.

[21] S. Kim, H. Lee, D. Kim et al., "Transparent conductive films of copper nanofiber network fabricated by electrospinning," Journal of Nanomaterials, vol. 2015, Article ID 518589, 8 pages, 2015.

[22] B. Hwang, T. Kim, and S. M. Han, "Compression and tension bending fatigue behavior of $\mathrm{Ag}$ nanowire network," Extreme Mechanics Letters, vol. 8, pp. 266-272, 2016.

[23] Y. Ahn, Y. Jeong, and Y. Lee, "Improved thermal oxidation stability of solution-processable silver nanowire transparent electrode by reduced graphene oxide," ACS Applied Materials and Interfaces, vol. 4, no. 12, pp. 6410-6414, 2012.

[24] B. Hwang, Y. An, H. Lee et al., "Highly flexible and transparent ag nanowire electrode encapsulated with ultra-thin $\mathrm{Al}_{2} \mathrm{O}_{3}$ : thermal, ambient, and mechanical stabilities," Scientific Reports, vol. 7, article 41336, 2017.

[25] S. Chen, L. Song, Z. Tao et al., "Neutral-pH PEDOT:PSS as over-coating layer for stable silver nanowire flexible transparent conductive films," Organic Electronics, vol. 15, no. 12, pp. 36543659, 2014.

[26] Y.-S. Liu, J. Feng, X.-L. Ou, H.-F. Cui, M. Xu, and H.-B. Sun, "Ultrasmooth, highly conductive and transparent PEDOT:PSS/ silver nanowire composite electrode for flexible organic lightemitting devices," Organic Electronics: Physics, Materials, Applications, vol. 31, pp. 247-252, 2016.

[27] C. Mayousse, C. Celle, A. Carella, and J.-P. Simonato, "Synthesis and purification of long copper nanowires. Application to high 
performance flexible transparent electrodes with and without PEDOT:PSS," Nano Research, vol. 7, no. 3, pp. 315-324, 2014.

[28] C.-K. Cho, W.-J. Hwang, K. Eun, S.-H. Choa, S.-I. Na, and H.-K. Kim, "Mechanical flexibility of transparent PEDOT:PSS electrodes prepared by gravure printing for flexible organic solar cells," Solar Energy Materials and Solar Cells, vol. 95, no. 12, pp. 3269-3275, 2011.

[29] C. Teng, X. Lu, Y. Zhu, M. Wan, and L. Jiang, "Polymer in situ embedding for highly flexible, stretchable and water stable PEDOT:PSS composite conductors," RSC Advances, vol. 3, no. 20, pp. 7219-7223, 2013. 

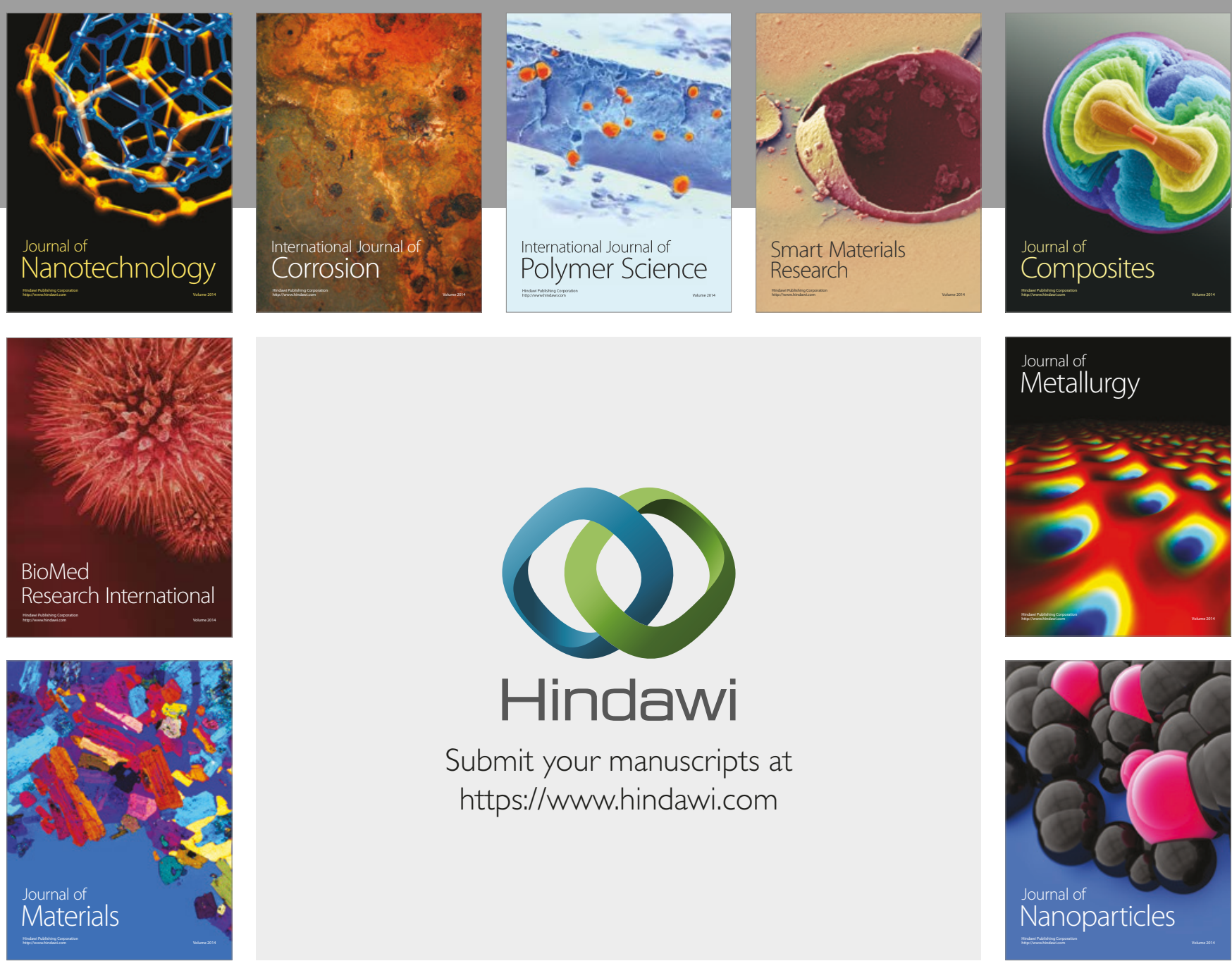

\section{Hindawi}

Submit your manuscripts at

https://www.hindawi.com
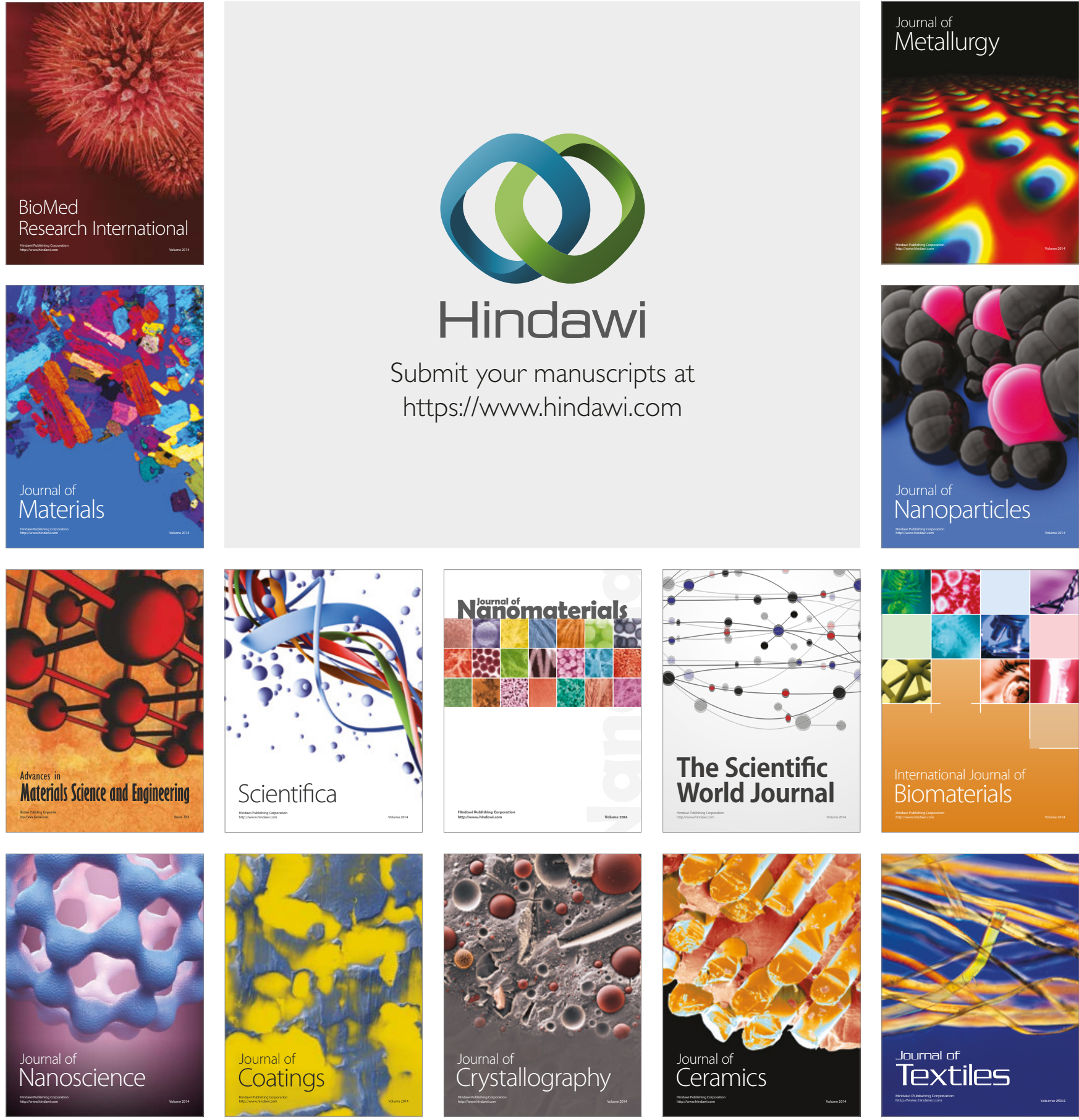

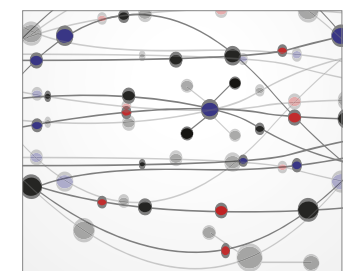

The Scientific World Journal
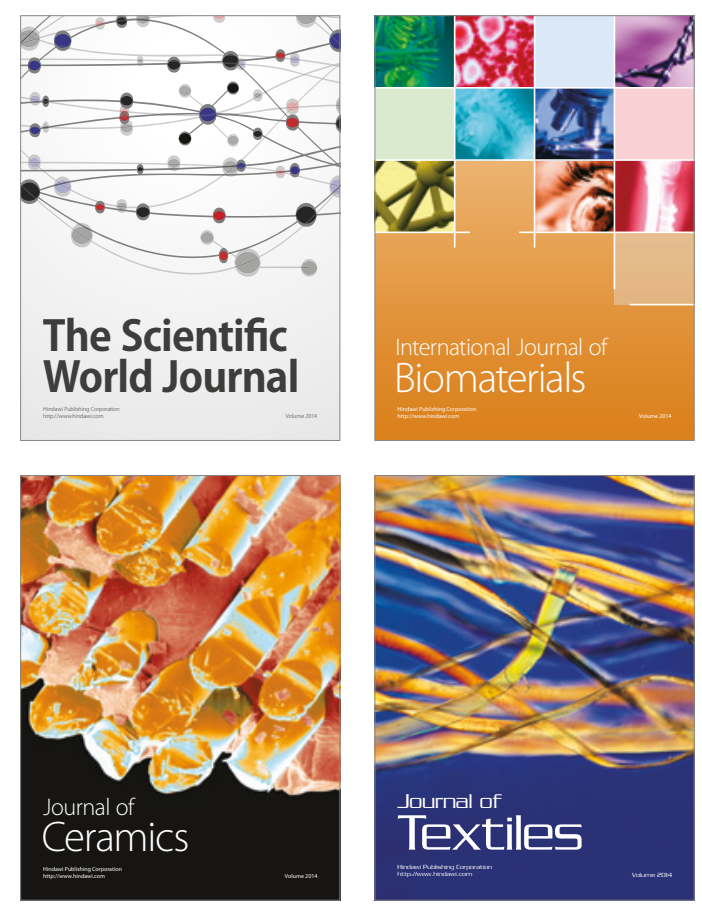